\title{
LA GESTIÓN PÚBLICA DEL TURISMO EN EL DISTRITO METROPOLITANO DE CARACAS. FORTALEZAS, DEBILIDADES Y NUEVOS DESAFÍOS.
}

\author{
Autor \\ Sandra Ornés Vásquez. \\ Urbanista \\ Master en Gerencia Empresarial \\ Diplomada en Políticas Públicas y en Gestión Municipal del Turismo. \\ Doctoranda en Desarrollo Sostenible por la USB. \\ Departamento de Planificación Urbana \\ Universidad Simón Bolívar (USB) \\ Venezuela \\ sornes@usb.ve
}

\section{RESUMEN}

La presente investigación tiene como finalidad identificar las fortalezas y debilidades del proceso de gestión pública del turismo del Distrito Metropolitano de Caracas, a la luz de activar aquellos cambios que sean necesarios para lograr su potenciación desde la óptica del turismo urbano, del desarrollo urbano local y el fortalecimiento del proceso de descentralización venezolano. Para ello, se desarrolló una investigación de tipo exploratoria-descriptiva, con bases teóricas en las áreas de gerencia, ciudad, planificación, gestión pública y turismo; y una metodología fundamentada en la recolección y registro sistemático de la información; proceso que permite identificar coincidencias entre los diferentes actores locales para la construcción de una visión compartida de la ciudad y proponer una nueva estrategia de gestión turística fundamentada en la planificación, el desarrollo social y económico, la inversión y proyectos, y el mercadeo; a ser institucionalizada en todos los niveles del gobierno (local, regional y nacional).

Palabras claves: turismo metropolitano, gestión turística, gestión de ciudades. 


\title{
PUBLIC MANAGEMENT OF IN THE METROPOLITAN DISTRICT OF CARACAS: STRENGTHS, WEAKNESSES AND NEW CHALLENGES.
}

\author{
Autor \\ Sandra Ornés Vásquez. \\ Urbanista \\ Master en Gerencia Empresarial \\ Diplomada en Políticas Públicas y en Gestión Municipal del Turismo. \\ Doctoranda en Desarrollo Sostenible por la USB. \\ Departamento de Planificación Urbana \\ Universidad Simón Bolívar (USB) \\ Venezuela \\ sornes@usb.ve
}

\begin{abstract}
This research aims to identify the strengths and weaknesses of the management of tourism in the Metropolitan District of Caracas, in order to activate those changes necessary to achieve their full potential in terms of urban tourism, local urban development and strengthening of the decentralization process in Venezuela. For this purpose, an exploratory and descriptive-type research was developed, with theoretical foundations in the areas of management, city planning, public management and tourism, and a methodology based on the systematic collection and recording of information, process that helps to identify matches among different local actors in order to build a shared vision of the city and to propose a new management strategy based on tourism planning, social and economic development, investment and projects, and marketing, to be institutionalized at all government levels (local, regional and national).
\end{abstract}

Key words: metropolitan tourism, tourist management, management of cities. 


\section{INTRODUCCIÓN.}

El turismo constituye una actividad integral e integradora, factor clave en cualquier estrategia de desarrollo y crecimiento, de acuerdo a datos manejados por la Organización de Estados Americanos (OEA). En América Latina, el 6\% del total del empleo corresponde a las actividades turísticas (Olivier, 1998-1999: 7), y en este contexto, de acuerdo a la Organización Mundial del Turismo (OMT), el franco crecimiento de esta actividad deberá ser absorbido por las ciudades.

Ante tal compromiso, las ciudades deben tener la capacidad de responder a las expectativas y necesidades de los turistas, así como renovar y mejorar las facilidades y/o atractivos, de manera de mantener su participación en el competitivo mercado del turismo y los beneficios que resultan de éste.

Así mismo, la Ciudad y su gobierno deben asegurarse que dicha actividad económica sea desarrollada y gestionada beneficiando a la población local, contribuyendo al mejoramiento del ambiente urbano, y al fortalecimiento de la capacidad financiera de la autoridad local; transformándose así en una gran oportunidad.

En consecuencia, si se parte del concepto de Ciudad como un organismo vivo y dinámico, un sistema funcional, que aglomera un conjunto de componentes relacionados e interdependientes, entonces resulta fundamental coordinar y gestionar estos componentes sobre la base de una visión compartida por todos los actores involucrados en el turismo urbano para el logro de la ciudad que se desea. Por ello, si se quiere promover una ciudad turística, es importante definir con claridad: ¿Cuál es el mensaje/ imagen que se desea dar?; ¿Cuáles serán las premisas que sustenten su desarrollo? ; ¿Cómo establecer una efectiva coordinación entre los distintos gobiernos locales que integran las Áreas Metropolitanas?.

En el caso Venezolano, a pesar de los grandes esfuerzos realizados a partir de las políticas nacionales, regionales y locales implantadas, el Turismo se ha transformado en una "actividad incontrolada, con acciones anárquicas, sin coordinación institucional" e inter-institucional (Briceño, 1989: 582). De allí radica la preocupación por lograr una ordenación, coordinación y gestión pública del espacio urbano y la actividad turística en la ciudad de Caracas, bajo un criterio de ciudad sustentable, a partir de la identificación de las fortalezas y debilidades de la gestión pública del turismo en el Distrito Metropolitano de Caracas.

Una vez asumido este enfoque, resulta necesario establecer lineamientos estratégicos, metodologías y líneas de acción para que los gobiernos locales generen un 
cambio de paradigma en la prestación del servicio turístico por parte del sector público; y de esta manera superar el divorcio existente entre la Planificación y la Acción.

Igualmente, a partir de los resultados de la presente investigación, se asomará la necesaria definición y formulación de una propuesta para coordinación metropolitana de la gestión pública del turismo, que gestione, controle y oriente el aprovechamiento del capital turístico de la capital venezolana.

\section{METODOLOGÍA}

La presente investigación se caracteriza por ser de tipo exploratorio-descriptivo, sobre la base de un enfoque explicativo y un diseño no experimental, la cual incluyó: 1) una revisión documental y bibliográfica amplia en materia de ciudad, turismo, gerencia y gestión pública, así como aspectos legales, a los fines de sistematizar la información e identificar los puntos de confluencia, y 2) la aplicación de cuestionarios y entrevistas estructurados a fuentes primarias vinculadas con el turismo en los cinco municipios que conforman el Distrito Metropolitano de Caracas, éstos orientados de la siguiente manera:

- Cuestionarios: buscan construir el diagnostico de la situación del turismo local, los intereses de los actores involucrados y la percepción de la ciudad como destino turístico. Estos fueron aplicados a las dependencias de turismo municipal (Oficinas o Unidades), los representantes del sector privado del turismo (AVAVIT Y FENAHOVEN), y a los turistas extranjeros presentes en el Aeropuerto Internacional de Maiquetía, que conocieran la ciudad de Caracas. Adicionalmente se aplico una consulta adicional a loa alcaldes electos en el año 2001, a propósito de su visión sobre el turismo local y la ciudad.

- Entrevistas: se focaliza hacia el conocimiento de las tendencias del turismo local, su visión en el contexto regional y nacional, y posibles acciones para su fortalecimiento. Estas entrevistas fueron aplicadas a representantes de los entes nacionales y regionales del turismo (Ministerio de Turismo, Corporaciones Regionales y Fundapatrimonio), así como a representantes de fundaciones u organizaciones no gubernamentales y académicas con inherencia en los temas de turismo, ciudad y gestión local. (Fundacomún, CLAD, ILDIS, Fundación de Gerencia Social, IESA, Fundación Plan Estratégico de Caracas Metropolitana, CENDES).

A partir de los datos recolectados, se realizó el correspondiente procesamiento y análisis, con la finalidad de identificar ejes y posiciones frente a la gestión turística del 
Distrito Metropolitano de Caracas, tanto coincidentes como divergentes, siendo estos la base para la definición de los nuevos desafíos a establecer en esta materia.

Finalmente, a los efectos contextualizar la realidad identificada en esta investigación, presentar los resultados, y derivar las correspondientes conclusiones, el presente artículo tratará en primer lugar las principales características del área de estudio (Distrito Metropolitano de Caracas), posteriormente se paseará por un marco teórico y referencial (nacional e internacional) de las gerencia, el turismo y la ciudad, que busca explicar la complejidad urbana orientada hacia el turismo; luego se presentarán los resultados de la aplicación de los instrumentos ya señalados; y finalmente se plantea tanto las conclusiones como los aportes relacionados con la definición de una nueva estrategia de coordinación para la gestión turística para la ciudad.

\section{DESCRIPCIÓN DEL ÁREA DE ESTUDIO.}

El Distrito Metropolitano de Caracas (ciudad de Caracas) se encuentra localizado al norte y centro de Venezuela y se define como la principal aglomeración urbana y capital de Estado. Posee una población estimada para los años 2000 y 2010 de 3.223.000 y 3.580.000 habitantes respectivamente (Fundación Plan Estratégico de Caracas Metropolitana (FPECM): 1998), lo cual representa alrededor del $12 \%$ del total de la población venezolana.

Desde el punto de vista de su distribución territorial, posee una extensión aproximada de 81.790 has, parte de las cuales también forman parte de la Zona Protectora del Área Metropolitana (843.000 has); y está integrada por cinco (5) Municipios: uno (1) correspondiente al Distrito Capital (Municipio Libertador) y cuatro (4) integrantes del Estado Miranda (Municipios Baruta, El Hatillo, Chacao y Sucre).

Dentro de "un sistema de ciudades demográficamente bastante equilibrado y bien distribuido en el territorio" (Fundación Plan Estratégico Caracas Metropolitana, 1998: $\mathrm{s} / \mathrm{p}$ ), la ciudad de Caracas es una metrópolis concentradora de actividades prestadoras de servicios, que está en constante transformación, integradora de múltiples actores que participan en la dinámica urbana y se constituye en el principal centro político y económico de Venezuela.

Desde el punto de vista de su gobernabilidad, el Distrito Metropolitano de Caracas ha perdido unicidad política al estar conformada por cinco (5) municipios y sobre ella sobreponerse competencias nacionales, regionales y locales. En cuanto a la gestión de la ciudad (administración, planificación y financiamiento), resulta evidente la falta de coordinación y de definición de criterios orientadores, condición que se refleja en el desmejoramiento de los niveles de cobertura y calidad de los servicios públicos 
fundamentales (equipamientos urbanos, de red, vialidad y transporte) y el ordenamiento territorial; ya sea por falta de mantenimiento o limitaciones en la ejecución de nuevas inversiones.

En cuanto a su economía, la metrópolis caraqueña se orienta hacia la actividad terciaria, y específicamente hacia las ramas del comercio, la construcción, los servicios y el sector público; albergando el 18\% de la población ocupada en la rama económica de los Servicios a nivel nacional, y el 13\% de las actividades comerciales, de la construcción y el sector público (GRUSON, 2006). Sin embargo, igual que en el resto del país, se evidencian problemas tales como: pobreza, marginalidad, exclusión social, e inseguridad; ésta última común en las grandes ciudades.

Dado este panorama, es conveniente y urgente ofrecerle al Distrito Metropolitano de Caracas la posibilidad de autogestionarse, de coordinarse, de ser productiva y competitiva, integradora socialmente, aprovechando su potencial físico-ambiental, productivo y humano, además de constituirse y reconocerse como la puerta de entrada de Venezuela.

Dentro de una realidad socio-política donde el proceso de descentralización y autonomía local es un hecho, a pesar de las amenazas centralizadoras de la política nacional actual; las herramientas gerenciales pueden apoyar y ser aplicadas al entorno municipal y metropolitano, en busca de la eficiencia, la transparencia y la racionalización de los procesos vinculados con la gestión municipal y la gestión turística. De esta manera, se fomentaría que dicha gestión sea fundamentalmente estratégica, descentralizada, participativa, eficaz, concertadora de los intereses de los actores involucrados, y coordinadora del logro de los objetivos del Municipio y la Ciudad, en torno a la actividad turística.

\section{MARCO TEÓRICO Y REFERENCIAL}

\section{La Gerencia y la Ciudad}

La evolución de las ciudades ha estado marcada por los avances que en materia social y económica fundamentalmente éstas han podido lograr, pasando de la época rural, la industrial, hasta la contemporánea o moderna, la cual no solo evidencia cada día mas una concentración de la población en áreas urbanas con el consecuente intercambio cultural, sino que también la ciudad es influenciada por una fuerte tendencia hacia la dispersión o relocalización de los procesos productivos en los espacios urbanos y hacia la integración global de la dirección y la gestión de los mismos. 
Si se parte del concepto de ciudad como un complejo ecosistema integrado por elementos o partes conectadas, donde las actividades humanas están enlazadas por comunicaciones que interactúan en la medida que dicho sistema evoluciona y se transforma (Teoría General de Sistemas), entonces se podría afirmar que dichos componentes deben estar focalizados hacia la consecución de unos fines comunes (Fernández, 1997: 61).

Este fin debe ser la necesidad de implementar "estrategias inteligentes y factibles de inserción en la economía global que responda simultáneamente a las necesidades del mercado y de sus habitantes", que en el caso de las ciudades son los ciudadanos (Fundación Plan Estratégico Caracas Metropolitana, 1998: 12); donde las mismas deben ser implantadas desde la Gestión Pública, entendida como:

- "Entramando de reglas e incentivos a través de las que el Estado elabora y ejecuta las políticas públicas”.

- "Disposiciones institucionales que orienten el comportamiento de los actores internos y externos a una mayor racionalidad económica". (Spink et al, 2001: 101 y 103).

Para ello, es fundamental involucrar variables tales como: gobierno, manejo de recursos, estructuras organizativas, políticas públicas, etc.; lo cual demanda la búsqueda de mecanismos que garanticen la eficiencia del sector público, bajo criterios de responsabilidad, compromiso y resultados, en beneficio de la comunidad y del desarrollo local, cuya responsabilidad recae en los gobiernos locales que integran las principales ciudades.

Dada la complejidad de este compromiso, el gerente municipal o local debe asumir una actitud de estratega, de planificador, condición que implica la aproximación a las condiciones más favorables, mientras se evalúa el momento más oportuno para actuar en función de una realidad conocida y un compromiso claro.

Dentro de este marco conceptual, evidentemente el planificador gubernamental debe prepararse para entender el comportamiento del entorno y prever las posibles consecuencias que se puedan generar sobre el espacio urbano. En este contexto, Fernández (1997: 51-52), realiza algunas afirmaciones que perfectamente son aplicables a la realidad venezolana:

- La descentralización de las competencias urbanísticas, ha favorecido la adaptación de la legislación urbanística a la peculiaridades de cada región, debilitando la planificación normativa y centralizada. (Esto a pesar de las actuales presiones provenientes desde el gobierno local, en busca de un retroceso hacia la centralización).

- Es evidente la irrupción de los agentes de desarrollo económico en las decisiones urbanísticas, ya sea públicos como privados. 
- Existe un creciente peso de los movimientos sociales en el urbanismo, ya que la sociedad civil comienza a intervenir, participar y comprometerse en el proceso de desarrollo urbano.

- La incorporación de innovaciones tecnológicas a la gestión urbanística, tales como los sistemas de información geográfica y la automatización de los procedimientos administrativos, ha facilitado la toma de decisiones ante la complejidad urbana.

- La insistente exigencia de transparencia gubernamental por parte de los ciudadanos, es un reflejo de la oposición asumida frente a proceso de planificación urbana realizados exclusivamente por técnicos y grupos económicos, a puertas cerradas.

Como consecuencia de estos planteamiento, surge la definición consensuada de la ciudad como el primer paso hacia una nueva forma de gestión local, a los fines de establecer objetivos multi-sectoriales vinculados con su ordenación, crecimiento y desarrollo, y permitir la participación decidida y comprometida de los agentes sociales y del ciudadano común para el logro de esta meta en pro de su futuro.

Al trasladar esta definición y nueva forma de gestión local a aquellas ciudades que cuentan con potencial turístico, se pueden identificar distintos momentos históricos que evidencian una constante búsqueda hacia un modelo de gestión del desarrollo. En la década de los años 60, principalmente en Europa, el enfoque urbanístico dió importancia a las variable física, el análisis y la evaluación detallada de los recursos turísticos existentes; concretándose en un plan de desarrollo físico denominado plan maestro. Sin embargo, al final de esta época, surge el enfoque de política económica, centrado en la aplicación de instrumentos que fomentaran el turismo y coadyuvaran al logro de los objetivos económicos y sociales planteados por el Estado. Ante estos avances, la década de los años 70 se constituyó en la etapa promotora de la integración de la actividad del turismo en el desarrollo económico, social y de ordenamiento territorial del país como un todo.

Aunque éstos representan valiosos esfuerzos para la definición y entendimiento del ámbito de la planificación turística, la misma aún está marcada por la tendencia hacia el aspecto físico, no contemplando las variables legales, organizacionales, financieras, y operacionales. En este sentido, cobra fuerza el tratamiento de la planificación de la actividad turística desde la base de la planificación estratégica, complementada con una planificación operativa, a los fines de lograr el desarrollo integral y armónico de esta actividad en áreas metropolitanas, haciéndolas competitivas.

"Las nuevas características y condiciones de la economía mundial le otorgan a las grandes ciudades un rol tan relevante en materia de apoyo a la productividad, que hace que el destino de las economías nacionales esté fuertemente asociado a la competitividad de aquellas" (Fundación Plan Estratégico Caracas Metropolitana, 1997-1998: 34). 
Es necesario que las ciudades y sus gobernantes comprendan que dentro de la nueva economía mundial, la competencia entre las ciudades es legítima, a los fines que las mismas se posicionen como importantes comercializadoras de sus productos (la infraestructura, las atracciones, la imagen y calidad de vida, y la gente), respetando sus principios de valoración propia, evitando su estancamiento. "Si un lugar puede, primero debe arreglar sus bases (la infraestructura), añadir algunos atractivos, elevar la amistad y especialización de su gente, y después, promover su imagen precisa" (Kotler et al, 1994: 39). De esta manera, las ciudades podrán estar en condiciones de ser competitivas.

\section{La Planificación Urbanística y el Turismo.}

Al hablar de la planificación urbanística venezolana, se debe hacer referencia inmediatamente a las principales referencias nacionales y metropolitanas vigentes, como lo son: El Plan Nacional de Ordenación del Territorio y El Plan de Ordenación Urbanística del Área Metropolitana de Caracas, respectivamente, aunque éste último solo fue llevado a proyecto sin aprobación.

Estos planes superiores coinciden en reconocer la necesidad de consolidar un aparato productivo competitivo, global, en constante proceso de innovación y mejoramiento, con la decidida incorporación del sector privado, bajo la premisas fundamentales de: 1) expansión de la actividad económica hacia sectores de exportación no petroleros y petroleros; 2) el incremento de la inversión pública, 3) la recuperación del ahorro público, 4) la superación del déficit fiscal, y 5) la concentración de la inversión privada hacia ramas especializadas como: las telecomunicaciones, la industria petrolera, petroquímica y minera, el turismo, etc.( (Kotler et al, 1994: 87). En torno a ésta última actividad se plantea la búsqueda de un ordenamiento urbanístico y ambiental a favor del turismo, siempre y cuando se evite el desorden urbanístico, el deterioro ambiental y la contaminación.

Tradicionalmente la implementación de estrategias de planificación turística ha estado íntimamente vinculada con la delegación de competencias a nivel local y comunitaria, como principales actores involucrados en la toma de decisiones, con lo cual se reconocen las fortalezas del proceso de descentralización y los beneficios logrados hasta el día de hoy, a pesar del surgimiento de nuevas líneas centralistas desarrolladas y con pretensión de ser implementadas a nivel nacional. Sin embargo, el marco referencial por excelencia utilizado por cada una de las regiones y municipios del país lo constituyen los Planes Nacionales de Turismo, entre los que se mencionan: VI Plan del Sector Turismo, realizado por Corpoturismo en el año 1979, el IX Plan de Turismo del ano 1995, el Plan de Desarrollo Turístico para el período 1995-1999, y más recientemente el proyecto de Plan Nacional Estratégico de Turismo 2007-2012, el cual define al turismo como una 
prioridad nacional dada su importancia como factor de desarrollo y motor de crecimiento, que permite y facilita la inclusión social para el desarrollo sustentable y sostenible.

Sin embargo, a nivel municipal y metropolitano no se ha avanzado tanto. Sólo destaca, en el área de estudio, el esfuerzo realizado por la Gobernación del Estado Miranda, a través de la Corporación Mirandina de Turismo (Corpomitur), la cual elaboró en el año 1999, el Plan de Turismo del Estado, y cuyas directrices pueden ser aplicadas en los municipios Baruta, Chacao, Sucre y El Hatillo del Distrito Metropolitano de Caracas. El mismo considera la región metropolitana como un multidestino turístico y de recreación, caracterizado por poseer una extraordinaria localización, diversidad de sus atractivos turísticos, y contener a una importante parte del principal núcleo urbano emisor del país.

En consecuencia, constituye un imperativo el desarrollo de planes turísticos que se correspondan con las premisas de los Planes de Desarrollo Urbano Local (PDUL) de cada uno de los Municipios integrantes del Distrito Metropolitano de Caracas, así como futuros planes estratégicos que se consideren pertinentes aprobar a nivel Metropolitano.

En este contexto, se puede destacar que algunos de los mayores avances a favor de los gobiernos locales, se centran: en lo político, al permitir una mayor identidad entre los ciudadanos y sus gobernantes, del mandatario y su institución, y la aparición de nuevos liderazgos; en lo económico, al avizorar fuerzas productivas nacientes y con propuestas innovadoras; y en la capacidad de gestión, al canalizar la búsqueda de respuestas ante los problemas de orden social, desarrollo productivo, seguridad ciudadana, recreación, etc. (Mascareño, 2000: 4):

Reconociendo estos avances de los gobiernos locales y potenciando el valor turístico que representa el Distrito Metropolitano de Caracas, es necesario lograr la alianza y compromiso entre: el gobierno, en términos de lograr la integración del turismo a la política del Estado y la puesta en práctica de incentivos a la actividad turística; la sociedad y sector publico-privado, en torno a la planificación y desarrollo de la infraestructura básica, a largo plazo.

Sin embargo, aunque la ciudad pueda tener atractivos y ventajas que la hagan potencialmente una oferta turística, evidencia plasmada en la captación de más del $30 \%$ del total de turistas extranjeros para el año 2007-2008, según estadísticas del Ministerio del Poder Popular para el turismo; se requiere de un adecuado plan y gestión del turismo y de desarrollo local para lograr su efectividad.

Este plan de gestión turística debe estar en sintonía con las principales referencias que marcan la tendencia mundial en el área, entre las que destacan: 
- La Carta del Turismo, acordada en la Asamblea General de la Organización Mundial del Turismo (1985) la cual recoge los principios y conceptos de Declaración de Manila sobre el Turismo Mundial (10-10-80), los Estatutos de la Organización Mundial del Turismo, la Resolución y recomendaciones adoptadas en la Conferencia de las Naciones Unidas sobre el Turismo y los Viajes Internacionales (09-63), y la Declaración Universal de los Derechos Humanos adoptada por la ONU (10-1248); en la cual considera al turismo como un derecho fundamental, y constituye un instrumento adecuado para mejorar la calidad de vida. Igualmente, destaca en su articulo III (de un total de nueve) relacionado con el papel de los Estados, que los mismos deberán favorecer el crecimiento ordenado y armonioso de la actividad turística, integrar su política turística en su política global de desarrollo a sus diversos niveles (local, regional, nacional e internacional), estimular la adopción de medidas que permitan la participación de todos en el turismo, y proteger el medio ambiente turístico.

- La Agenda 21 para los Viajes y El Turismo, enmarcada en el Programa 21 adoptado en la Conferencia de Naciones Unidas sobre el Medio Ambiente y el Desarrollo, en la Cumbre de la Tierra (1992), la cual define principios para la protección del futuro sostenible del planeta.

- La Declaración de Davos, aprobada en la II Conferencia Internacional sobre Cambio Climático y Turismo celebrada en el ano 2007, la cual hace un llamado para el logro de un mayor apoyo financiero y técnico a los destinos turísticos y operadores de los países en desarrollo, a los fines de garantizar que puedan participar en la respuesta global al cambio climático; dado que se reconoce que el turismo contribuye al calentamiento global y es, al mismo tiempo, víctima del calentamiento global.

Dentro de este contexto, resulta interesante conocer ¿cómo la normativa venezolana contribuye y acoge estos acuerdos, preocupaciones y propuestas?.

La referencia legal a considerar lo constituye la Ley Orgánica de Turismo, aprobada en fecha 23-06-05, bajo la Gaceta Oficial $N^{\circ} 38.215$, la cual tiene como objetivo "promover y regular la actividad turística como factor de desarrollo sustentable del país, mediante el establecimiento de normas que garanticen la orientación, facilitación, el fomento, la coordinación y el control de la actividad turística, estableciendo los mecanismos de participación y concertación de los sectores público y privado en esta actividad“ (ANRBV-LOT, 2005: art.1). Cabe destacar que con esta ley la actividad turística es declarada de utilidad pública y de interés general.

De esta forma, el papel fundamental de los entes de gobierno estadal y municipal pasa a ser el fomentar e integrar en los planes de desarrollo local de los diferentes niveles de gobierno, la actividad turística, a través de: 
- La formulación de los proyectos turísticos en su circunscripción, en coordinación con la Corporación de Turismo estadal y en concordancia con los lineamientos y políticas dictadas por el Ministerio de Turismo en el Plan Nacional Estratégico de Turismo.

- El desarrollo de los planes de ordenación del territorio, en el ámbito de sus competencias, conforme con el espacio turístico existente y con el Plan Nacional Estratégico de Turismo.

- La elaboración, actualización, publicación y difusión del inventario de atractivos turísticos, prestadores de servicios turísticos y el catálogo turístico municipal. (ANRBV-LOT, 2005: art.29).

Y especificando las competencias municipales, la Constitución de la República Bolivariana de Venezuela (CRBV, 1999), La Ley Orgánica de Ordenación Urbanística (LOOU, 1987), aún sometida a la discusión de su vigencia después de la derogatoria de la Ley Orgánica para la Planificación y Gestión de la Ordenación del Territorio (LOPGOT), La Ley Orgánica del Poder Público Municipal (LOPPM, 2005), y la Ley Especial sobre el Régimen del Distrito Metropolitano de Caracas (LERDMC, 2000), reconocen a los Municipios como, "la unidad política primaria de la organización nacional” (CRBV, 1999, art.168).....a los cuales les concierne todo lo que tenga que ver con la vida local, "en especial la ordenación y promoción del desarrollo económico y social.” (CRBV, 1999, art.178), sobre la base de la promoción de la participación y el mejoramiento de las condiciones de vida de la comunidad.

Sin embargo, a partir de la aprobación de la Ley Especial Distrito Metropolitano de Caracas, bajo la Gaceta Oficial No 36.906, de fecha 08-03-2000, la política pública metropolitana pasa a ser entendida como "un sistema de gobierno municipal a dos niveles: el primero denominado Nivel Metropolitano, formado por un órgano ejecutivo y un órgano legislativo, cuya jurisdicción comprende la totalidad territorial Metropolitana de Caracas; y el segundo, identificado como Nivel Municipal, formado por un órgano ejecutivo y un órgano legislativo en cada municipio integrante del Distrito Metropolitano de Caracas, con jurisdicción municipal” (ANRBV-LERDMC, 2000: art.3); condición que compromete la gobernabilidad de la ciudad, a la luz del cumplimiento de aquellas competencias concurrentes, en materia del desarrollo local, por parte de ambos niveles de gobierno dentro de la misma ciudad.

En consecuencia, es necesario avanzar en el reconocimiento de esta nueva figura de gobierno metropolitano como una oportunidad para el logro de una coordinación y planificación del turismo en el Distrito Metropolitano de Caracas, garantizando una mejor gestión pública en esta materia; esto a pesar que el resultado de los balances metropolitanos desde su creación no sean muy alentadores en cuanto al turismo urbano y la ciudad. Mas aún en los tiempos actuales de polarización política y de implantación 
de una nueva autoridad de gobierno con competencia sobre el Municipio Libertador o Distrito Capital, como territorio estratégico por la concentración de poderes nacionales; que por lo reciente de su creación no es incorporada en la presente sección.

\section{RESULTADOS DE LA INVESTIGACIÓN}

A partir de la aplicación de un total de seis (6) entrevistas a representantes del turismo nacional, estadal y local; doce (12) cuestionarios a los principales actores de la gestión turística municipal en Caracas; dos (2) cuestionarios de opinión a representantes del sector privado del turismo, y treinta y cinco (35) cuestionarios de opinión a turistas extranjeros, usuarios del Aeropuerto Internacional Simón Bolívar en Maiquetía; todos instrumentos orientados hacia el reconocimiento de los avances y debilidades de la gestión turística de la ciudad de Caracas, y las percepciones que de esta condición poseen cada uno de los actores que conforman la dinámica turística-urbana; se pudo evidenciar que existen componentes estructurales a considerar al momento de proponer cualquier estrategia de gestión publica del turismo en el Distrito Metropolitano de Caracas:

- Comunicación e Información: existe una inadecuada comunicación entre los actores públicos responsables, mostrando una actuación cortoplacista, con un enfoque sectorial, que deriva en una clara inexistencia de control y seguimiento de las acciones emprendidas para el logro de una visión metropolitana.(Cuadro $\mathrm{N}^{\circ} 1$ )

\section{Cuadro No 1}

Nivel de comunicación (coincidencia de respuestas) intermunicipal vinculada con la gestión turística.

\begin{tabular}{|c|c|c|c|}
\hline MUNICIPIOS & Preguntas Coincidentes & Total Preguntas & Índice de Coincidencia (\%) \\
\hline LIBERTADOR & 20 & 32 & 62,5 \\
\hline BARUTA & 12 & 32 & 37,5 \\
\hline CHACAO & 14 & 32 & 43,75 \\
\hline SUCRE & 7 & 32 & 21,875 \\
\hline EL HATILLO & 17 & 32 & 53,125 \\
\hline
\end{tabular}

Fuente: Ornés, Año 2001.

- Coordinación: los distintos municipios y el Estado de Miranda, no disponen de una estructura organizativa acorde e integrada con las demás dependencias involucradas en el acontecer turístico urbano.

- Políticas y Lineamientos: no existe una política turística clara para los municipios 
que integran la ciudad de Caracas; y las políticas o lineamientos rectores nacionales sólo se quedan a nivel del gobierno central, sin ser incorporados a la gestión gubernamental local.

- Marco Legal Vigente: ofrece grandes oportunidades a los gobiernos locales. Sin embargo, las autoridades locales no han incorporado dentro de sus prioridades la materia turística. Sólo el 40\% de los Municipios (Chacao y El Hatillo) manifestaron que el turismo se encontraba incluido dentro de la gestión local de sus municipios.

- Concepción de la Actividad Turística: el turismo se trata de una manera segmentada, considerándolo en un $40 \%$ como la variable de recreación, animación y ocio requerido por la población, y también como una actividad económica que genera beneficios; y en un $20 \%$ como actividad que fomenta valores culturales.

- Potencial Turístico de la Ciudad: existe coincidencia de opiniones entre los representantes municipales (80\%) en asegurar que la ciudad de Caracas posee un gran potencial para el desarrollo del turismo, fundamentado en la valorización patrimonial y escénica, la oferta de restaurantes, Cafes, Pubs, áreas comerciales en general y la organización de eventos culturales públicos (Cuadros $\mathrm{N}^{\circ} 2$ ). El sector privado, por su parte, considera en un $100 \%$ que la ciudad de Caracas si cuenta con un potencial y un atractivo turístico, y que los mismos se centran fundamentalmente en sus atractivos tradicionales e históricos. Sin embargo, estos representantes privados reconocen que a pesar de este potencial, el gobierno local no ha contribuido al desarrollo de la actividad turística de la ciudad. De hecho destacan que los aspectos más descuidados son: la seguridad, la limpieza (salubridad) y mantenimiento, y la señalización (Cuadro $\mathrm{N}^{\mathrm{o}} 3$ ). 


\section{Cuadro No 2}

Potencial turístico y atractivos de la ciudad de Caracas según representantes municipales.

\begin{tabular}{|l|c|c|}
\hline \multicolumn{1}{|c|}{ ATRACTIVO } & $\mathrm{N}^{\mathrm{o}}$ Municipios & Porcentaje (\%) \\
\hline Edificaciones con valor patrimonial & 4 & 80 \\
\hline Centros culturales y museos & 2 & 40 \\
\hline Centros de convenciones y conciertos & 3 & 60 \\
\hline Áreas recreacionales, plazas, paseos & 3 & 60 \\
\hline $\begin{array}{l}\text { Restaurantes, cafes, pubs y locales relacionados con } \\
\text { la gastronomía }\end{array}$ & 4 & 80 \\
\hline Parques nacionales & 3 & 60 \\
\hline Áreas comerciales variadas & 4 & 80 \\
\hline Infraestructura hotelera de primera & 3 & 60 \\
\hline $\begin{array}{l}\text { Organización de eventos culturales públicos } \\
\text { frecuentes. }\end{array}$ & 4 & 80 \\
\hline Otros & 1 & 20 \\
\hline
\end{tabular}

Fuente: Ornés, Año 2001.

\section{Cuadro No 3}

Aspectos que perjudican la actividad turística en la ciudad de caracas, según sector privado.

\begin{tabular}{|l|c|c|}
\hline \multicolumn{1}{|c|}{ ASPECTOS } & $\begin{array}{c}\mathrm{N}^{\circ} \text { representantes } \\
\text { privados }\end{array}$ & Porcentaje (\%) \\
\hline Seguridad & 2 & 100 \\
\hline Limpieza & 2 & 100 \\
\hline Señalización & 2 & 100 \\
\hline Respeto al turista & 1 & 50 \\
\hline Personal preparado & 1 & 50 \\
\hline La información & 1 & 50 \\
\hline Cultura de servicio & 1 & 50 \\
\hline
\end{tabular}

Fuente: Ornés, Año 2001.

Con respecto a los turistas extranjeros consultados, éstos opinan mayoritariamente que el potencial de la ciudad se centra en su valor ambiental (clima), la oferta de centros comerciantes y las opciones de diversión existentes. Sin embargo, aspectos desfavorables 
como la inseguridad ciudadana, el deterioro físico de la ciudad, la falta de señalización e información, y los limitados medios de transporte, entre otros; atentan contra las ventajas comparativas de la ciudad. (Cuadros $\mathrm{N}^{\circ} 4$ y 5).

\section{Cuadro No 4}

Percepción favorable de Caracas por parte de los turistas extranjeros entrevistados.

\begin{tabular}{|l|c|c|}
\hline \multicolumn{1}{|c|}{ CARACTERÍSTICAS } & $\mathrm{N}^{\text {o }}$ Turistas & Porcentaje (\%) \\
\hline Segura & 4 & 11,4 \\
\hline Amena y agradable & 19 & 54,3 \\
\hline Con opciones de diversión & 19 & 54,3 \\
\hline Posee buena señalización turística & 6 & 17,1 \\
\hline Cuenta con módulos de información al turista & 6 & 17,1 \\
\hline Clima Agradable & 28 & 80,0 \\
\hline Adecuado transporte y vialidad & 10 & 28,6 \\
\hline Limpia y cuidada & 3 & 8,6 \\
\hline Ofrece Espacios Públicos & 12 & 34,3 \\
\hline Ofrece buenos centros comerciales & 25 & 71,4 \\
\hline Ofrece eventos culturales variados & 15 & 42,9 \\
\hline Otros & 1 & 2,9 \\
\hline
\end{tabular}

Fuente: Ornés, Año 2001.

\section{Cuadro $\mathbf{N}^{\circ} 5$}

Percepción desfavorable de Caracas por parte de los turistas extranjeros entrevistados.

\begin{tabular}{|l|c|c|}
\hline \multicolumn{1}{|c|}{ CARACTERÍSTICAS } & No Turistas & Porcentaje (\%) \\
\hline Insegura & 26 & 74,3 \\
\hline Agresiva y aburrida & 12 & 34,3 \\
\hline Sin opciones de diversión & 5 & 14,3 \\
\hline No cuenta con señalización turística & 17 & 48,6 \\
\hline No se ofrece información al turista & 17 & 48,6 \\
\hline Clima Desagradable & 0 & 0,0 \\
\hline Deficiente transporte y vialidad & 15 & 42,9 \\
\hline Sucia y descuidada & 21 & 60,0 \\
\hline No cuenta con Espacios Públicos & 6 & 17,1 \\
\hline No ofrece buenos centros comerciales & 1 & 2,9 \\
\hline No tiene oferta cultural variada & 6 & 17,1 \\
\hline
\end{tabular}

Fuente: Ornés, Año 2001. 
En este contexto, el sector privado, de manera unánime, considera que la autoridad local debe canalizar sus esfuerzos de control y gestión urbana hacia la satisfacción de los servicios públicos, infraestructura para la movilidad vehicular y peatonal, la seguridad y la información hacia el turista; opinión que coincide con la apreciación aportada por los turistas extranjeros entrevistados a objeto de la presente investigación.

- Disposición de los Alcaldes (autoridad local): todos reconocen el potencial de desarrollo turístico de la ciudad, el $40 \%$ de ellos están dispuestos a apoyar las acciones de promoción y divulgación turística, mejoramiento de los espacios urbanos, y seguridad ciudadana; y el 20\% apoya la idea de promover incentivos impositivos (reducción o exoneración de impuestos) a favor de la actividad turística.

Esta disposición manifestada por los Alcaldes está en sintonía con las recomendaciones presentadas por los turistas extranjeros entrevistados en torno al Distrito Metropolitano de Caracas, ya que los mayores valores apuntan hacia la búsqueda de un mejor trato e información al turista (42,9\%), garantizar la seguridad pública (40\%), velar por el ornato urbano $(31,4 \%)$ y mejorar la vialidad y el tránsito $(25,7 \%)$. Sin embargo, el logro de este modelo de gobernabilidad urbana, en torno a la gestión del turismo, debe superar y consensuar la posición de los alcaldes en torno a la modalidad de dicha gestión, decidiendo entre el nivel local, el nivel metropolitano o el doble nivel; y cuyos resultados se presentan a continuación (Cuadro $\mathrm{N}^{\circ}$ 6):

\section{Cuadro No 6}

Opinión sobre la modalidad de gestión del turismo en el distrito metropolitano de Caracas *

\begin{tabular}{|c|c|c|c|}
\hline MUNICIPIO & MODALIDAD & RAZONES & Porcentaje (\%) \\
\hline Chacao & Doble Nivel & $\begin{array}{r}\text { Coordinación de la } \\
\text { Implementación de políticas } \\
\text { turísticas }\end{array}$ & $\mathbf{2 0}$ \\
\hline Chacao y Libertador & Doble Nivel / Local & $\begin{array}{c}\text { Realización de acciones concretas } \\
\text { a nivel local (descentralización) }\end{array}$ & $\mathbf{4 0}$ \\
\hline Metropolitano & Metropolitano & $\begin{array}{r}\text { Manejar la promoción y } \\
\text { negociación con los agentes del } \\
\text { mercado a escala }\end{array}$ & $\mathbf{2 0}$ \\
\hline Baruta & Doble Nivel & $\begin{array}{r}\text { Se logra el apoyo del gobierno a } \\
\text { varios niveles. } \\
\text { Está involucrado el compromiso } \\
\text { de los recursos locales }\end{array}$ & $\mathbf{2 0}$ \\
\hline
\end{tabular}




\begin{tabular}{|l|l|c|c|}
\hline Hatillo & Doble Nivel & $\begin{array}{c}\text { Constituye un factor de desarrollo } \\
\text { económico local. } \\
\text { El Municipio local es parte de la } \\
\text { oferta turística que es Caracas }\end{array}$ & $\mathbf{2 0}$ \\
\hline
\end{tabular}

Fuente: Ornés, Año 2001.

* El Municipio Sucre no emitió opinión.

Dado el potencial turístico de la ciudad de Caracas que los entes involucrados coinciden en señalar, y la escasa o deficiente capacidad de gestión que han demostrado los gobiernos locales que integran el espacio territorial de la ciudad de Caracas, constituye un verdadero reto lograr la coordinación y trabajo integrado de estos actores del sector público local como promotores del desarrollo local, de manera conjunta con el sector privado como grandes inversionistas, y la comunidad como motor dinamizador y beneficiario de la ciudad.

El desarrollo turístico en ciudades debe ser considerado como componente de la planificación y gestión urbana, siendo éstas responsabilidad de los gobiernos locales.

\section{CONCLUSIONES Y APORTES FINALES.}

Ante los resultados obtenidos, debe abogarse por la formulación de un Plan Estratégico de Turismo para el Distrito Metropolitano, que oriente las intervenciones tanto públicas de los cinco municipios de la ciudad de Caracas, como privadas, y reconozca al turismo mas allá del simple ocio o del evento puntual de mercadeo anual de la capital (por ejemplo, FITCAR). Así mismo, el impulso de la aplicación de principios de gerencia efectiva y asertiva, además de sustentable, donde los beneficios generados de esta actividad puedan permanecer en la ciudad y de los cuales podamos disfrutar todos sus habitantes.

En consecuencia, se justifica la búsqueda de una coordinación de la Gestión Pública del Turismo para Caracas, aplicable para cualquier Área Metropolitana; que se fundamenta en la visión compartida e imagen de ciudad (generación de producto), construida por los actores consultados y que se resume de la siguiente manera: “Caracas, ciudad cosmopolita, concentradora de actividades, servicios y valores culturales competitivos, que le ofrece al turista y al ciudadano un espacio con alto nivel de calidad urbana y ambiental para el intercambio, el encuentro, la memoria y el disfrute".

Para ello, debe definirse y construirse una nueva estructura o modelo de gestión en el cual se integren los sectores público, privado, y comunitarios; en torno al adecuado manejo de la imagen y calidad de vida de la ciudad, su infraestructura, sus atracciones y 
su gente. Este modelo debe soportarse en cuatro (4) componentes estratégicos, a partir de los cuales definirán las líneas de acción y prioridades:

a) Planificación, involucra el conocimiento y análisis de los recursos con que se cuenta (diagnóstico de fortalezas y debilidades), para así poder generar un producto turístico, que en este caso es la ciudad.

Responsables: oficinales municipales de catastro, planificación urbana, cultura, recreación y turismo, ambiente, seguridad ciudadana.

Actividades: inventario de atractivos turísticos, áreas recreacionales, espacios públicos, tradiciones y valores populares; identificación del estado de los servicios públicos y equipamientos; formulación de planes de desarrollo turístico y sus regulaciones a nivel urbano; gestión de áreas protegidas y parques urbanos; diseño de rutas turísticas, promoción de actividades culturales y de fortalecimiento de la identidad local; programas de educación ambiental; planes de protección, seguridad y vigilancia; sistemas de información y atención al turista y ciudadano.

b) Desarrollo Socio-económico, vinculado con las políticas de fomento empresarial sobre todo en el área turística, de producción de bienes y servicios, de seguridad social, de capacitación de recursos humanos y concientización ciudadana; las cuales permitan el sustento de la actividad turística en el Distrito Metropolitano.

Responsables: oficinas municipales de educación, desarrollo socio-económico, prensa y relaciones públicas.

Actividades: formación para el trabajo; programas de asesoramiento para la conformación de pequeñas y medianas empresas; programas de capacitación en el área del turismo; promoción de incentivos para el incremento en la producción de bienes y servicios; programas de educación y sensibilización hacia el turismo y la ciudad.

c) Inversiones y provectos, busca definir y acordar el tipo de inversiones públicas y privadas requeridas por la ciudad para el aprovechamiento de su potencial turístico, incluyendo las actuaciones urbanas vinculadas con la dotación de equipamientos, infraestructura y servicios; apoyándose en la creación de incentivos fiscales que se consideren pertinentes.

Responsables: oficinas municipales de infraestructura de red, vialidad y transporte, impuestos y tributos, y el sector privado.

Actividades: monitoreo de la calidad de los servicios de agua, cloacas, drenajes, telecomunicaciones, aseo urbano, etc.; mantenimiento y mejoramiento de la infraestructura vial y el transporte público; rescate y ampliación de espacios públicos; promoción de incentivos fiscales en materia turística y de acondicionamiento urbano; generación de nuevos atractivos a partir de proyectos de desarrollo urbano integrales; planes de eficiencia en los procesos administrativos; etc. 
d) Mercadeo, establece la política de información, comunicación y promoción del potencial turístico de la ciudad, a nivel nacional e internacional; la cual debe permanecer en continuo proceso de revisión y ajuste, en función de la propia dinámica del turismo urbano, las necesidades humanas y su gestión por parte de las autoridades locales, regionales y nacionales.

Responsables: oficinas municipales de prensa y relaciones publicas, Corpomitur, Ministerio de Turismo, oficina de turismo sector privado.

Actividades: política de información, comunicación y promoción de la imagen de la ciudad; apertura hacia la firma de convenios, organización de bolsas de turismo y eventos-encuentros en general, etc.; desarrollo de nuevos productos, a partir de la definición de mercados meta; disposición de módulos de información al turista (local y extranjero), incluyendo material impreso.

Cada uno de los componentes podrán plantearse organizacionalmente como comisiones integrantes de la Coordinación Metropolitana del Turismo del Distrito Metropolitano de Caracas, y deberán establecer sus lineamientos, principios y formas de actuación dentro de todo el sistema, teniendo como pivote o articulador a la dependencia responsable del turismo a nivel local. (Figura $\mathrm{N}^{\circ} 1$ ). Estos estarían respaldados por el marco legal turístico vigente que ofrece grandes oportunidades a los gobiernos locales en cuanto a su desarrollo a partir de la actividad turística, a lo cual se une el reconocimiento, por parte de los actores de la ciudad, del potencial de Caracas en esta rama económica. 
Figura $\mathbf{N}^{\circ} 1$

Modelo de coordinación de

la gestión turistica del distrito metropolitano de Caracas

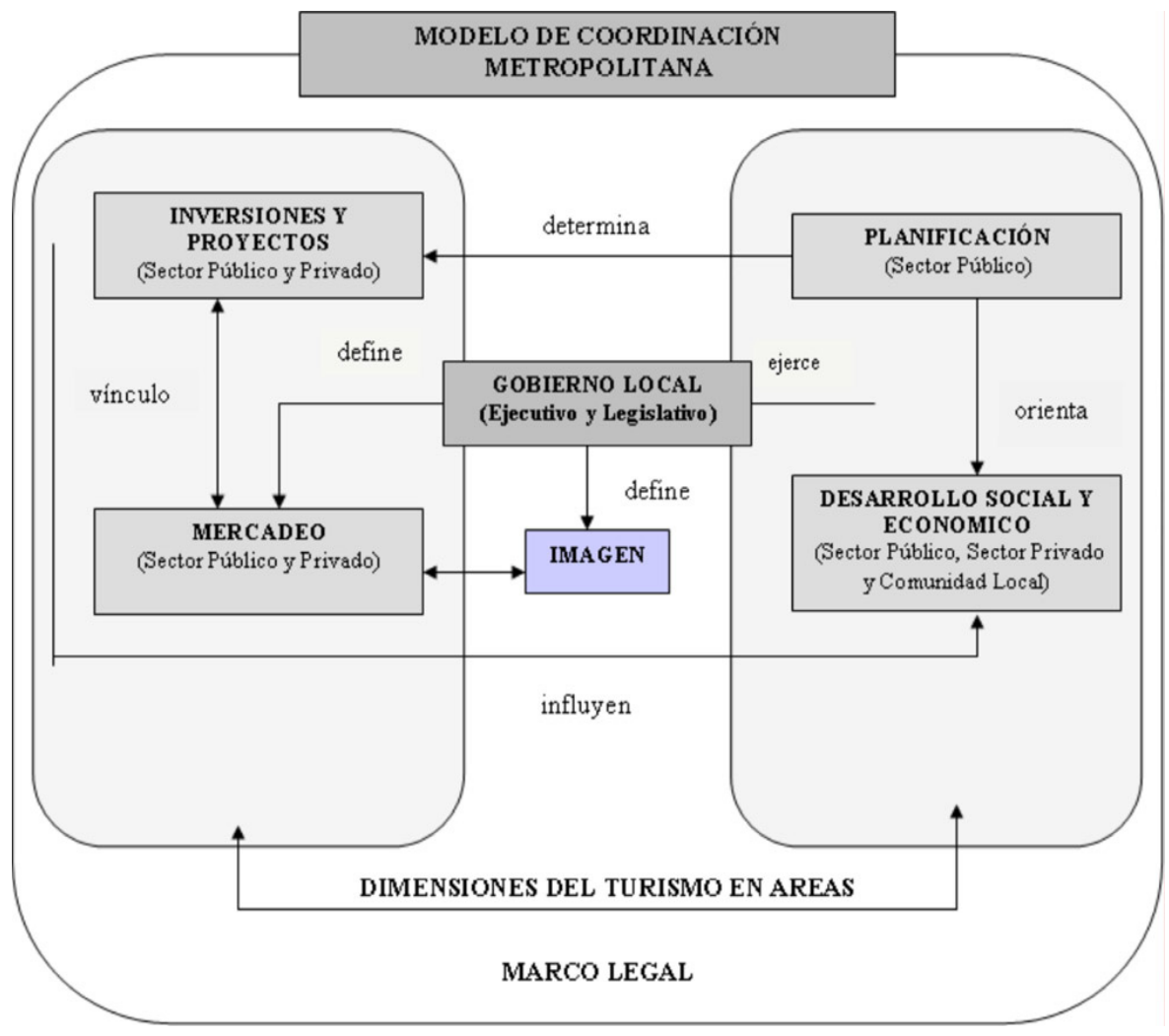

Fuente: Ornés, Año 2001.

De esta forma, se propone que este patrón sea asumido e institucionalizado a todos los niveles de gobierno (local, regional y nacional), a fin de garantizar la efectiva articulación entre todos los actores, la coherencia en la toma de decisiones, el adecuado uso de los recursos, y la gobernabilidad de la ciudad, bajo un criterio de competitividad y gestión eficaz.(Figura № 2). 


\section{Figura $\mathrm{N}^{\circ} 2$}

Institucionalización del modelo de coordinación metropolitana de la gestion turistica

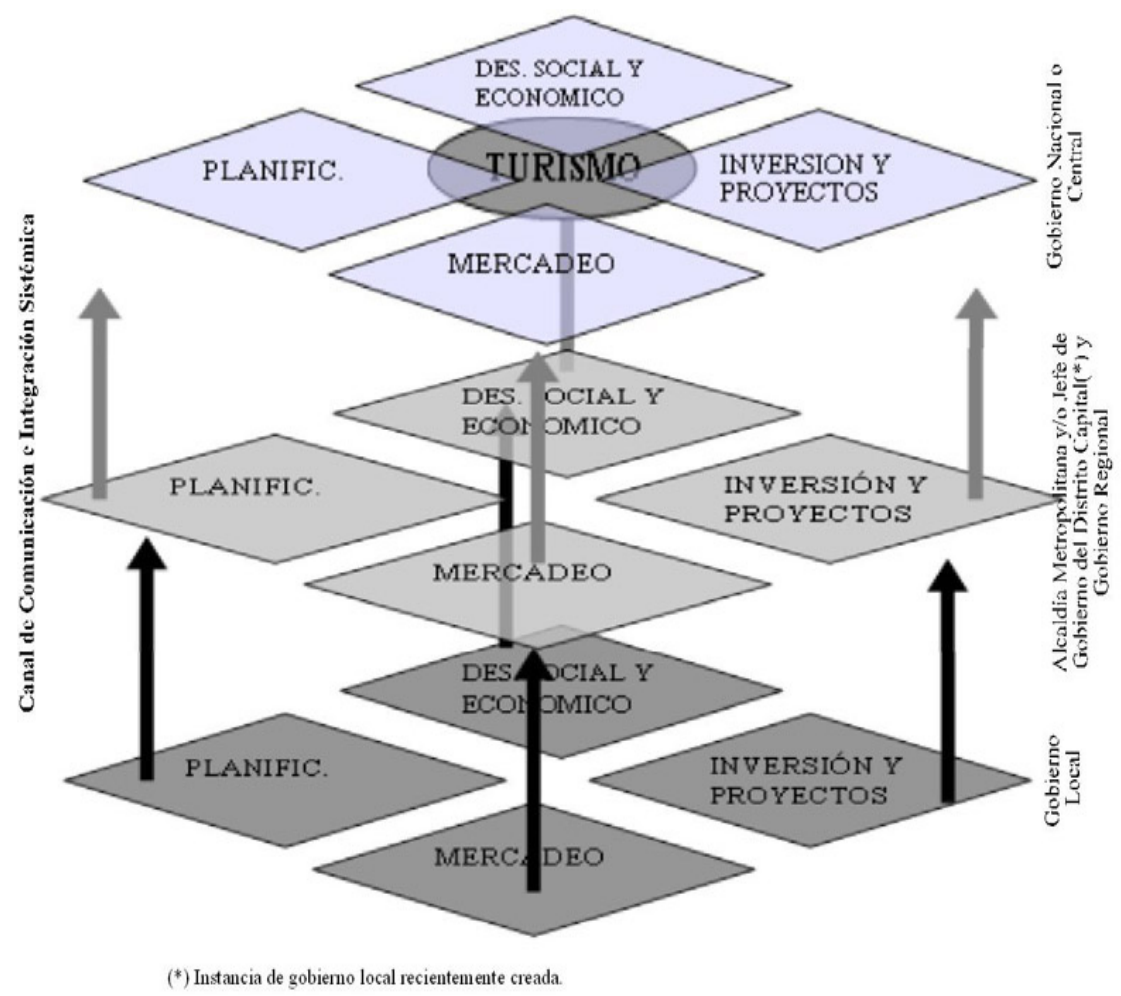

Fuente: Ornés, Año 2001.

Igualmente, se espera que este modelo:

1. Mejore e institucionalice los canales de comunicación e intercambio de información entre los actores vinculados al sector turístico.

2. Facilite la definición de una visión compartida de la ciudad, surgida de sus debilidades y fortalezas; en la cual participen todos los actores e intereses.

3. Promueva la coordinación intra e interinstitucional de las acciones vinculadas con el mejor aprovechamiento y gestión de los recursos destinados al desarrollo y promoción del turismo en la ciudad. 
4. Oriente la toma de decisiones hacia el establecimiento de políticas coherentes y factibles en materia turística y urbana.

Parte de la presente investigación fue presentada en la Conferencia Mundial sobre Desarrollo de Ciudades.

Porto Alegre-Brasil. 2008.

Recibido: 22/08/2009

Aprobado:09/11/2009

Arbitrado anonimamente

\section{REFERENCIAS BIBLIOGRÁFICAS.}

- Asamblea Nacional de La República Bolivariana de Venezuela (ANRBV) (2006): "Ley Orgánica para la Planificación y Gestión de la Ordenación del Territorio (LOPGOT)”. Gaceta Oficial N 38.388. Venezuela. (Derogada al día siguiente de su promulgación).

- Asamblea Nacional de La República Bolivariana de Venezuela (ANRBV) (2005): “Ley Orgánica del Poder Público Municipal (LOPPM)". Gaceta Oficial N 38.204. Venezuela.

- Asamblea Nacional de La República Bolivariana de Venezuela (ANRBV) (2005): “Ley Orgánica de Turismo (LOT)". Gaceta Oficial No 38.215. Venezuela.

- Asamblea Nacional de La República Bolivariana de Venezuela (ANRBV) (1999): “Constitución de la República Bolivariana de Venezuela (CRBV)”. Gaceta Oficial No 36.860 . Venezuela.

- Asamblea Nacional de La República Bolivariana de Venezuela (ANRBV) (2000): "Ley Especial sobre el Régimen del Distrito Metropolitano de Caracas (LERDMC)". Gaceta Oficial No 36.906. Venezuela.

- Briceño F., Frank (1989): "Hacia una nueva estrategia en materia de turismo". en: El Rol del Estado Venezolano en una nueva estrategia económica. CaracasVenezuela. COPRE.

- Congreso Nacional de la República de Venezuela (1987): "Ley Orgánica de Ordenación Urbanística”. Gaceta Oficial Nº 33.868. Venezuela.. 
- Fernández G., José M. (1997): “Planificación Estratégica de Ciudades”. BarcelonaEspaña. Edit. Gustavo Gili S.A. Serie: Proyecto de Gestión.

- Fundación Plan Estratégico Caracas Metropolitana (FPECM) (1997-1998): "Plan Estratégico Caracas Metrópoli 2010. Una Propuesta para la ciudad”. Papeles de trabajo e informes de los resultados de las mesas de trabajo. Caracas-Venezuela.

- Fundación Plan Estratégico Caracas Metropolitana (FPECM) (1998): “Caracas, Presente y Futuro. Ideas para transformar la ciudad". Papel de trabajo enmarcado en el "Plan Estratégico Caracas Metrópoli 2010”. Caracas-Venezuela.

- Gruson, Alberto (2006): "Morfología del Empleo. Venezuela 1995-2005”. CaracasVenezuela. Edit. CISOR-Centro de Investigación Social.

- Kotler, Philip et al (1994): "Mercadotecnia de Localidades". México. Edit. Diana.

- Mascareño, Carlos (2000): “Gestión Local”, en "Modernización de las Gobernaciones en Venezuela. Logros y Tendencias". Caracas-Venezuela. Edit. PNUD/Banco Mundial/ILDIS/ Gobernación del Zulia/G-Local.

- Olivier U., Lidia (1998-1999): “Estudio sobre el Turismo y el Empleo en Venezuela”. Caracas-Venezuela. Edit. OIT-ONU.

- Ornés, Sandra (2001): "Propuesta de un Sistema Metropolitano de Gestión Pública del Turismo". Caracas-Venezuela. Facultad de Ciencias Económicas y SocialesUniversidad Central de Venezuela. Tesis de Maestría.

- Spink, Peter; et al (2001): "Nueva Gestión Pública y Regulación en América Latina. Balances y Desafíos". Caracas-Venezuela. Edit. CLAD-BID. 